\title{
Glutamine modulates acute dextran sulphate sodium-induced changes in small-intestinal intraepithelial $\gamma \delta$-T-lymphocyte expression in mice
}

\author{
Man-Hui Pai ${ }^{1}$, Jun-Jen Liu ${ }^{2}$, Sung-Ling Yeh ${ }^{3}$, Wei-Jao Chen ${ }^{4}$ and Chiu-Li Yeh ${ }^{5 *}$ \\ ${ }^{1}$ Department of Anatomy, Taipei Medical University, Taipei, Taiwan, ROC \\ ${ }^{2}$ School of Medical Laboratory Science and Biotechnology, Taipei Medical University, Taipei, Taiwan, ROC \\ ${ }^{3}$ School of Nutrition and Health Sciences, Taipei Medical University, Taipei, Taiwan, ROC \\ ${ }^{4}$ Department of Surgery, College of Medicine, National Taiwan University, Taipei, Taiwan, ROC \\ ${ }^{5}$ Department of Food and Nutrition, Chinese Culture University, 55, Hwa-Kang Road, Yang-Ming-Shan, Taipei 11114, \\ Taiwan, $R O C$ \\ (Submitted 3 April 2013 - Final revision received 27 June 2013 - Accepted 29 July 2013 - First published online 14 November 2013)
}

\begin{abstract}
The present study investigated the effect of glutamine (Gln) on dextran sulphate sodium (DSS)-induced changes in the expression of small-intestinal intraepithelial lymphocyte (IEL) $\gamma \delta$-T cells in mice. Mice were randomly assigned to a normal control (NC) group and two DSS-treated groups. The NC group and one of the DSS-treated groups (DSS-C) were fed a common semi-purified diet, while the other DSS-treated group (DSS-G) was fed an identical diet, except that part of casein was replaced by Gln, which provided $25 \%$ of total amino acid nitrogen. After being fed the diets for $10 \mathrm{~d}$, mice in the NC group were given distilled water, while the DSS-treated groups were given distilled water containing $2.5 \%$ DSS for $5 \mathrm{~d}$. At the end of the experiment, the mice were killed. The small-intestinal IEL $\gamma \delta$-T-cell subset was isolated for further analysis. The results indicated that DSS treatment resulted in a lower percentage of small-intestinal IEL $\gamma \delta$-T cells and higher mRNA expressions of interferon- $\gamma$, TNF- $\alpha$, IL-17, complement 5 a receptor and keratinocyte growth factor in IEL $\gamma \delta-T$ cells. Gln administration increased the proportion of small-intestinal IEL $\gamma \delta$-T cells, and the expression levels of immunomodulatory mediator genes in IEL $\gamma \delta$-T cells were lower in the DSS-treated mice. The histological findings indicated that the immunoreactive intensity of the tight junction protein ZO-1 in the small-intestinal mucosa was higher in the DSS-G group than in the DSS-C group. These results indicate that pretreatment with Gln increases the proportion of small-intestinal IEL $\gamma \delta$-T cells and down-regulates $\gamma \delta$-T-cell-expressed inflammatory mediators, which may consequently ameliorate the severity of DSS-induced small-intestinal epithelial injury.
\end{abstract}

\section{Key words: Glutamine: Dextran sulphate sodium: Small-intestinal intraepithelial lymphocytes: $\gamma \delta$-T cells: Inflammatory} mediators: ZO-1 tight junction protein

Inflammatory bowel disease (IBD), which includes Crohn's disease and ulcerative colitis, refers to chronic inflammatory disorders that may affect the entire gastrointestinal $\operatorname{tract}^{(1)}$. The precise aetiology of IBD remains unidentified ${ }^{(2)}$. However, several factors including genetic predisposition, host immune system and intestinal microenvironment have been proposed to make major contributions to the pathogenesis of this disease ${ }^{(1)}$. Intestinal homeostasis depends on the dynamic crosstalk among the microbiota, intestinal epithelium and local immune cells ${ }^{(2)}$. Dysregulation or breakdown of the interactions between them may precipitate the intestinal inflammation observed in IBD.
The intestinal epithelium is lined by a single layer of epithelial cells that forms a physical barrier and is crucial for intestinal homeostasis. Small-intestinal intraepithelial lymphocytes (IEL) are a unique population of $\mathrm{T}$ cells that reside in the paracellular space between the epithelial cells. IEL serve as the first line of immune defence against invading pathogens and preserve the integrity of the mucosal barrier ${ }^{(3)}$. Gut IEL include two phenotypically distinct populations: conventional IEL, which express T-cell receptor (TCR) $\alpha \beta$ and either CO-receptor CD 4 or $\mathrm{CD} 8 \alpha \beta$ heterodimers, and unconventional IEL, which express either TCR $\gamma \delta$ or TCR $\alpha \beta$ and bear CD $8 \alpha \alpha^{+}$co-receptor molecules ${ }^{(4)}$. Small-intestinal IEL include

Abbreviations: C5aR, complement 5 a receptor; DSS, dextran sodium sulphate; DSS-C, dextran sulphate sodium-treated group fed the control diet; DSS-G, dextran sulphate sodium-treated group fed the glutamine diet; IBD, inflammatory bowel disease; IEL, intraepithelial lymphocytes; IFN, interferon; KGF, keratinocyte growth factor; NC, normal control group; RegIII $\gamma$, regenerating islet-derived protein III- $\gamma$; RPMI, Roswell Park Memorial Institute; TCR, T-cell receptor; TJ, tight junction.

* Corresponding author: C.-L. Yeh, email m8707008@hotmail.com 
a large proportion of $\gamma \delta$-T cells with a predominant $\mathrm{CD} 4^{-} \mathrm{CD} 8^{+}$ phenotype, and most of them express a homodimeric form of the CD $8 \alpha \alpha^{+}$co-receptor molecule ${ }^{(5)} \cdot \gamma \delta$-IEL help preserve the integrity of a damaged epithelial surface by facilitating the localised delivery of an epithelial cell growth factor ${ }^{(6)}$. The depletion of colonic $\gamma \delta$-T cells aggravates dextran sodium sulphate (DSS)-induced colitis. The protective effect of $\gamma \delta$-T cells has been confirmed in various IBD animal models ${ }^{(7)}$.

Glutamine (Gln) is the most abundant free amino acid in the mammalian plasma and intracellular pool. Gln has various physiological functions. It is essential for the proliferative response of enterocytes and rapidly dividing immune cells ${ }^{(8)}$. Numerous studies have shown that Gln has immunomodulatory properties. Previous studies have found that Gln supplementation enhances stress protein responses, attenuates inflammatory reactions, improves tissue metabolic functions and reduces oxidative stress under catabolic conditions ${ }^{(9)}$. A study carried out by Nose et al. ${ }^{(10)}$ has shown that Gln prevents total parenteral nutrition-associated changes in the phenotype and function of small-intestinal IEL and preserves the function of the epithelial barrier. Our previous study has also revealed that Gln administration prevents the apoptosis of smallintestinal IEL $\gamma \delta$-T cells and down-regulates $\gamma \delta$-T-cell-expressed inflammatory mediators in septic mice ${ }^{(11)}$. The DSS colitis murine model is usually used to assess novel treatments for $\mathrm{IBD}^{(12)}$. DSS-induced damage is not restricted to the colon; there are also morphological and biochemical changes throughout the small-intestinal mucosa ${ }^{(13)}$. To date, no study has investigated the effects of Gln on small-intestinal IEL $\gamma \delta$-T-cell subset distribution and subsequent inflammatory mediator changes in IBD. We hypothesised that Gln increases the proportion of cells of the TCR $\gamma \delta$ subset of small-intestinal IEL and down-regulates the expressions of inflammation-associated mediators, which may attenuate DSS-induced small-intestinal mucosal injury. Therefore, in the present study, we examined the expressions of genes involved in inflammatory responses and in the maintenance of small-intestinal epithelial tissue integrity in DSS-treated mice.

\section{Materials and methods}

\section{Animals}

Male C57BL/6 mice aged 8-12 weeks and weighing 22-25g at the beginning of the experiment were used in the present study. All the mice were housed in cages in a temperatureand humidity-controlled room and were allowed free access to a standard chow diet for 1 week before the start of the experiment. The mice were cared for in full compliance with the Guide for the Care and Use of Laboratory Animals (National Research Council, 1996), and protocols were approved by the Institutional Animal Care and Use Committee of Taipei Medical University.

\section{Experimental procedures}

A total of twenty-four mice were assigned to one normal control (NC) group and two DSS-treated groups, with each group having eight mice. The NC group and one of the DSS-treated groups (DSS-C) were fed a common semi-purified diet, while the other DSS-treated group (DSS-G) was fed an identical diet, except that part of casein was replaced by Gln, which provided $25 \%$ of total amino acid nitrogen (Table 1). The amount of Gln used is known to have an immunomodulatory effect on catabolic conditions ${ }^{(14)}$. The diets were fed for $10 \mathrm{~d}$ during the experimental period. Mice in the NC group were given distilled water, whereas the DSS-treated groups were given distilled water containing $2.5 \%(\mathrm{w} / \mathrm{v})$ DSS (molecular weight $40 \mathrm{kDa}$; MP Biomedicals) during the last $5 \mathrm{~d}$ (on days 6-10) of the experiment. During the experimental period, the body weight of the mice was recorded daily. At the end of the experiment (day 11), the mice were anaesthetised using an intraperitoneal Zoletil $(20 \mathrm{mg} / \mathrm{kg})$ injection and killed by cardiac puncture. To obtain peritoneal lavage fluid, $2 \mathrm{ml}$ of sterile saline were injected into the peritoneal cavity of the mice. Peritoneal lavage fluid samples were stored at $-80^{\circ} \mathrm{C}$ until inflammatory cytokine analysis. Small-intestinal tissue samples were collected for a histological examination. The small-intestinal IEL $\gamma \delta$-T-cell subset was prepared for gene expression analysis.

\section{Measurements of the concentrations of inflammatory cytokines in peritoneal lavage fluid}

The concentrations of IL-1 $\beta$, TNF- $\alpha$, IL- 6 and macrophage chemoattractant-1 in peritoneal lavage fluid were measured using ELISA kits according to the manufacturer's instructions (eBioscience).

\section{Preparation of small-intestinal intraepithelial lymphocytes}

Small-intestinal IEL were isolated as described previously, with some modifications ${ }^{(15)}$. After the removal of Peyer's patches and mesenteric fat tissue, the small intestine was cut

Table 1. Composition of the experimental diets $(\mathrm{g} / \mathrm{kg})$

\begin{tabular}{lcc}
\hline Components & Control diet & Gln diet \\
\hline Soyabean oil & 100 & 100 \\
Casein & 200 & 150 \\
Gln & 0 & $41 \cdot 7$ \\
Salt mixture & 35 & 35 \\
Vitamin mixture & 10 & 10 \\
Methyl cellulose & 31 & 31 \\
Choline bitartrate & 2.5 & 2.5 \\
Met & 3 & 3 \\
Maize starch & 626.8 & 618.5 \\
\hline
\end{tabular}

* The salt mixture contained the following $(\mathrm{mg} / \mathrm{g})$ : calcium phosphate dibasic, $500 ; \mathrm{NaCl}, 74$; potassium sulphate, 52; potassium citrate monohydrate, 20; magnesium oxide, 24; manganese carbonate, 3.5 ; ferric citrate, 6 ; zinc carbonate, 1.6; cupric carbonate, 0.3 ; potassium iodate, 0.01 ; sodium selenite, 0.01; chromium potassium sulphate, 0.55 .

tThe vitamin mixture contained the following $(\mathrm{mg} / \mathrm{g})$ : thiamin hydrochloride, 0.6 ; riboflavin, 0.6 ; pyridoxine hydrochloride, 0.7; nicotinic acid, 3; calcium pantothenate, 1.6; D-biotin, 0.05; cyanocobalamin, 0.001; retinyl palmitate, 1.6; DL- $\alpha$-tocopherol acetate, 20; cholecalciferol, 0.25 ; menaquinone, 0.005 . 
longitudinally, and the contents were flushed out with cold $\mathrm{Ca}-\mathrm{Mg}$-free PBS $\left(\mathrm{Ca}^{2+}, \mathrm{Mg}^{2+}\right.$-free PBS with $1 \mathrm{mM}$-HEPES, $2.5 \mathrm{~mm}-\mathrm{NaHCO}_{3}$ and $2 \%$ fetal bovine serum at $\mathrm{pH} 7 \cdot 3$ ). Later, the small intestine was cut into $5 \mathrm{~mm}$ pieces. Tissue pieces were placed in $20 \mathrm{ml}$ of Roswell Park Memorial Institute (RPMI) 1640 medium with 10\% fetal bovine serum and incubated for $30 \mathrm{~min}$ at $37^{\circ} \mathrm{C}$ with gentle shaking. The tissue pieces and medium were transferred into a $50 \mathrm{ml}$ centrifuge tube and vortexed at the maximum setting for $15 \mathrm{~s}$, and the supernatant was discarded. This step was repeated twice using fresh medium each time, and cells in the supernatants from each treatment were pooled. The supernatants were pooled and filtered through a $40 \mu \mathrm{m}$ nylon mesh, pelleted at $1600 \mathrm{rpm}$ for $10 \mathrm{~min}$ and resuspended in $40 \%$ Percoll (GE Healthcare) in RPMI 1640 medium. This cell suspension was overlaid with $70 \%$ Percoll in RPMI 1640 medium and centrifuged at room temperature and $2800 \mathrm{rpm}$ for $20 \mathrm{~min}$ without interruption. Small-intestinal IEL were recovered from the $40 \% / 70 \%$ interphase and washed twice with RPMI 1640 medium.

\section{Flow cytometric analysis}

CD103 is an integrin expressed by IEL. CD103 mediates the adhesion of lymphocytes to epithelial cells by interacting with their epithelium-specific ligands. To determine the percentages of $\gamma \delta$-T cells ${ }^{+}$belonging to the small-intestinal IEL population, the co-expression of $\mathrm{CD}_{103^{+}}$and $\mathrm{CD} 8 \alpha \alpha^{+}$by $\gamma \delta$-T cells was measured. A total of one million small-intestinal IEL were suspended in $100 \mu \mathrm{l}$ of staining buffer. IEL were incubated with purified rat anti-mouse CD16/CD32 (2.4G2; BD Bioscience) at $4^{\circ} \mathrm{C}$ for $10 \mathrm{~min}$ followed by staining with Pacific Blue anti-mouse CD103 (2E7; Biolegend), allophycocyanin anti-mouse TCR $\gamma / \delta$ (GL3; Biolegend), phycoerythrin antimouse $\operatorname{CD} 8 \alpha$ (53-6.7; Biolegend) and fluorescein isothiocyanate anti-mouse CD8 $\beta$ (YTS156.7.7; Biolegend) at $4^{\circ} \mathrm{C}$ for $15 \mathrm{~min}$. After two washes, the cells were studied using a BD FACSCantoII flow cytometer (BD Bioscience).

\section{Isolation of total small-intestinal intraepithelial lymphocyte $\gamma \delta$-T cells}

To study gene expressions in $\gamma \delta$-T cells, total small-intestinal IEL were stained with allophycocyanin anti-mouse TCR $\gamma / \delta$ (GL3; Biolegend), phycoerythrin anti-mouse CD8 $\alpha$ (53-6.7; Biolegend) and fluorescein isothiocyanate anti-mouse CD8 $\beta$ (YTS156.7.7; Biolegend). Small-intestinal IEL were purified on a cell sorter (BD FACSAria III; BD Biosciences). The $\mathrm{TCR}^{+} / \mathrm{CD} 8 \beta^{-}$subsets were isolated from $\gamma \delta$-T cells, and the purity ( $\geq 98 \%$ ) was checked.

\section{mRNA extraction and quantitative real-time $R T P C R$ analysis}

Total RNA was isolated from the purified small-intestinal $\gamma \delta$-IEL using the RNeasy Mini Kit (QIAGEN). The RNA pellet was dissolved in RNase-free water. The total RNA solution was stored at $-80^{\circ} \mathrm{C}$ for subsequent assays. The concentration of RNA was determined and quantified by measuring absorbance at 260 and $280 \mathrm{~nm}$ on a spectrophotometer. Complementary DNA was prepared from total RNA using the RevertAid ${ }^{\mathrm{TM}}$ First Strand cDNA Synthesis Kit (Fermentas) according to standard protocols. RT was carried out by subsequent incubation for $5 \mathrm{~min}$ at $65^{\circ} \mathrm{C}, 60 \mathrm{~min}$ at $42^{\circ} \mathrm{C}$ and $5 \mathrm{~min}$ at $70^{\circ} \mathrm{C}$. Complementary DNA was stored at $-80^{\circ} \mathrm{C}$ until use. Specific mRNA genes were amplified by real-time RT-PCR using the 7300 Real-Time PCR System (Applied Biosystems) with SYBR Green I as the detection format. The primers of the housekeeping gene ( $\beta$-actin), TNF- $\alpha$, interferon- $\gamma(I F N-\gamma), I L-4, I L-17$, complement 5 a receptor $(C 5 a R)$, keratinocyte growth factor $(K G F)$ and regenerating islet-derived protein III- $\gamma$ (RegIII $\gamma$ ) were purchased from Mission Biotech based on the deposited complementary DNA sequences (GenBank database, NCBI). The sequences of primers used for the quantitative RT-PCR assays are listed in Table 2. Amplification was carried out in a total volume of $25 \mu \mathrm{l}$ containing $1 \times$ Power SYBR Green PCR Master Mix (Applied Biosystems), $400 \mathrm{~nm}$ of each primer and $100 \mathrm{ng}$ of complementary DNA. The reaction was carried out using one cycle of $2 \mathrm{~min}$ at $50^{\circ} \mathrm{C}$ and $10 \mathrm{~min}$ at $95^{\circ} \mathrm{C}$, followed by forty cycles of $15 \mathrm{~s}$ at $95^{\circ} \mathrm{C}$ and $1 \mathrm{~min}$ at $60^{\circ} \mathrm{C}$, with a final dissociation curve analysis. Expression levels were quantified in duplicate by means of a real-time RT-PCR. Cycle threshold $\left(C_{\mathrm{t}}\right)$ values for the genes of interest were normalised to that of mouse $\beta$-actin and were used to calculate the relative mRNA expression levels.

\section{Histopathological and immunohistological examinations}

The middle segments of small-intestinal tissues were collected and fixed with $4 \%$ buffered paraformaldehyde overnight. The small-intestinal tissue samples were embedded in paraffin blocks (Leica Instruments). The small-intestinal tissue blocks were sectioned into $5 \mu \mathrm{m}$-thick sections and mounted on glass slides. To detect the integrity of tight junctions (TJ), TJ protein ZO-1 immunohistochemical staining was carried out.

Table 2. Sequences of primers used for inflammatory mediator quantitative real-time PCR assays

\begin{tabular}{lll}
\hline Accession no. & Gene name & $5^{\prime}-3^{\prime}$ primer sequence \\
\hline NM_007393.3 & $\beta$-Actin & F: ACCCACACTGTGCCCATCTAC \\
& & R: TCGGTGAGGATCTTCATGAGGTA \\
NM_013693.2 & TNF- $\alpha$ & $\begin{array}{l}\text { F: CCCTCACACTCAGATCATCTTCT } \\
\text { R: GCTACGACGTGGGCTACAG }\end{array}$ \\
NM_008337.3 & IFN- $\gamma$ & $\begin{array}{l}\text { F: ATGAACGCTACACACTGCATC } \\
\text { R: CCATCCTTTGCCAGTTCCTC }\end{array}$ \\
NM_021283.2 & IL-4 & F: ACAGGAGAAGGGACGCCAT \\
NM_010552.3 & IL-17 & F: AGCACCCTACAGACGAGCTCA \\
& & R: CATCTTCTCGACCCTGAAAGTGA \\
NM_007577.4 & C5aR & F: GAAGCGGCAACCTGGGGATGT \\
& R: CGTCTGGCTCGAAGGCTGTCAC \\
Z22703.1 & KGF & F: CGCAAATGGATACTGACACG \\
NM_011260.1 & RegIII $\gamma$ & F: GGGCTGGAACAGTTCACACT \\
& & R: CATCCACCTCTGTTGGGTTCA \\
\hline
\end{tabular}

$\mathrm{F}$, forward primer; $\mathrm{R}$, reverse primer; IFN- $\gamma$, interferon- $\gamma$; C5aR, C5a receptor; $K G F$, keratinocyte growth factor; Reglll $\gamma$, regenerating islet-derived protein III- $\gamma$. 
The tissue sections were deparaffinised and rehydrated with xylene and graded alcohol. After the retrieval of antigen with $10 \mathrm{~mm}$-citrate buffer ( $\mathrm{pH}$ 6.0), the sections were incubated with a primary antibody against ZO-1 (1:300; Abcam) overnight at $4{ }^{\circ} \mathrm{C}$, followed by incubation with a biotinylated secondary antibody (dilution 1:300; Chemicon) for $1 \mathrm{~h}$ at room temperature. After carrying out the reaction with the peroxidase-linked avidin-biotin complex (Vector) for $1 \mathrm{~h}$ at room temperature, a diaminobenzidine solution kit (Vector) was used to detect ZO-1 immunoreactivity. Haematoxylin (Sigma) nuclear staining was carried out to contrast-stain cell nuclei and cytoplasm. Digital images at $100 \times$ and $200 \times$ magnifications per section were captured with a Zeiss Axiophot light microscope equipped with a digital camera (Carl Zeiss). All the tissue sections were covered with cover slips using Permount (Fisher Scientific) and measured using the digital image analysis system (Image Pro Plus 5.1, Media Cybernetics). The 'count/size' and 'area' commands were used to determine the intensity of ZO-1 immunoreactivity. The automatic object counting and measuring processes were used to quantify the immunoreactive areas in the sections. Values are expressed as $\mu \mathrm{m}^{2}$. At least eight microscopic fields per section and three independent samples for each group were analysed, and averaged areas were obtained for each group.

\section{Statistical analysis}

All the data are expressed as means and standard deviations. Differences among the groups were analysed by a one-way ANOVA using Tukey's multiple-comparison test. A $P$ value $<0.05$ was considered to be statistically significant.

\section{Results}

Percentages of $\mathrm{CD} 103^{+} / T$-cell receptor $\gamma \delta^{+} / \mathrm{CD} 8 \alpha \alpha^{+}$ $T$ cells in small-intestinal intraepithelial lymphocytes

Compared with the NC group, the DSS-treated groups had lower percentages of small-intestinal IEL $\mathrm{CD} 103^{+} / \mathrm{TCR} \gamma \delta^{+} /$ $\mathrm{CD} 8 \alpha \alpha^{+} \mathrm{T}$ cells. The percentages of $\mathrm{CD} 103^{+} / \mathrm{TCR} \gamma \delta^{+} /$ $\mathrm{CD} 8 \alpha \alpha^{+} \mathrm{T}$ cells belonging to the small-intestinal IEL population were significantly higher in the DSS-G group than in the DSS-C group at the end of the experiment (Fig. 1).

\section{Concentrations of cytokines in peritoneal lavage fluid}

The concentrations of IL- $1 \beta$, IL- 6 , TNF- $\alpha$ and macrophage chemoattractant- 1 were significantly higher in the peritoneal lavage fluid of the DSS-C group than in that of the other two groups. The DSS-G group had lower concentrations of cytokines than the DSS-C group, and it exhibited no difference when compared with the NC group (Table 3).

\section{Expressions of proinflammatory mediator mRNA in small-intestinal $\gamma \delta$-intraepithelial lymphocytes}

The expression levels of $T N F-\alpha, I F N-\gamma, I L-17$ and $C 5 a R$ genes were higher in the DSS-C group than in the NC and DSS-G groups. There were no differences in the expression levels of these genes between the NC and DSS-G groups (Fig. 2).

Expressions of keratinocyte growth factor-1 and regenerating islet-derived protein III- $\gamma$ genes in small-intestinal $\gamma \delta$-intraepithelial lymphocytes

The expression of $K G F-1$ mRNA was significantly higher in the DSS-C group than in the NC and DSS-G groups. There were no differences in the expression of RegIII $\gamma$ gene between the NC and DSS-treated groups (Fig. 3).

Histopathological aspects and tight junction protein ZO-1 distribution in the small-intestinal mucosa

The expression of ZO-1 in the small-intestinal mucosa was examined by immunohistochemistry, which revealed that the epithelial structure differed among the distinct groups. In the NC group, the epithelium of the small-intestinal mucosa was intact. The small-intestinal mucosa of the DSS-C group exhibited villus denudation, subepithelial space formation and epithelial lifting. A less extensive ZO-1 distribution was also observed in the DSS-C group. The extent of smallintestinal epithelial damage was less severe and the expression of ZO-1 was more obvious in the DSS-G group than in the DSS-C group. The representative images obtained for each group are shown in Fig. 4(a) (left: 100X magnification of images; right: 200× magnification of the images). The quantification of ZO-1-immunoreactive areas among the groups is shown in Fig. 4(b). The immunoreactive areas of the DSS-G and NC groups were significantly larger than those of the DSS-C group. No differences in ZO-1-immunoreactive areas were found between the NC and DSS-G groups.

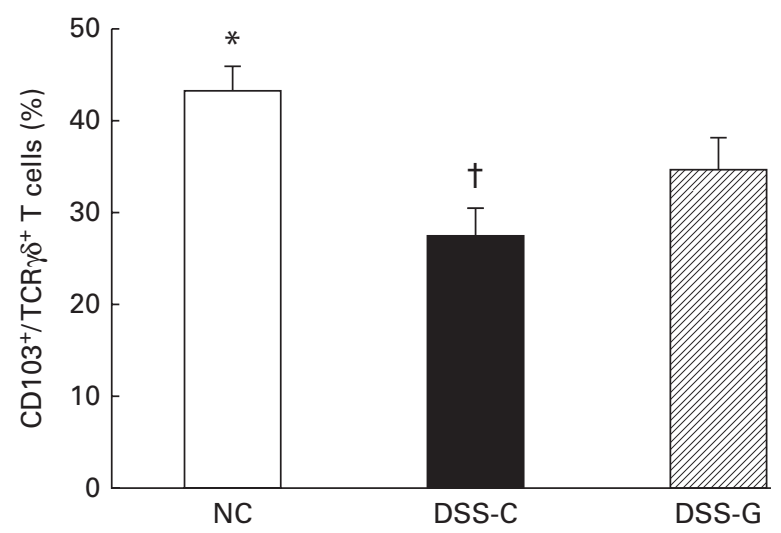

Fig. 1. Distributions of $\gamma \delta-T$ cells in small-intestinal intraepithelial lymphocytes in the normal control (NC) group, dextran sulphate sodium (DSS)-treated group fed the control diet (DSS-C) and DSS-treated group fed the glutamine diet (DSS-G) for $10 \mathrm{~d}$ and given DSS water for $5 \mathrm{~d}$. All the data are representative of duplicate measurements $(n 6)$. Values are means, with standard deviations represented by vertical bars. Differences among the groups were analysed by a one-way ANOVA with Tukey's multiplecomparison test. ${ }^{*}$ Mean value was significantly different from those of the other two groups $(P<0.05)$. † Mean value was significantly different from that of the DSS-G group $(P<0.05)$. 
Table 3. IL-1, IL-6, TNF- $\alpha$ and macrophage chemoattractant protein-1 (MCP-1) concentrations in peritoneal lavage fluid $\dagger$

(Mean values and standard deviations)

\begin{tabular}{|c|c|c|c|c|c|c|c|c|}
\hline & \multicolumn{2}{|c|}{ IL-1 $1 \beta$} & \multicolumn{2}{|c|}{ IL-6 (pg/ml) } & \multicolumn{2}{|c|}{$\mathrm{MCP}-1$} & \multicolumn{2}{|c|}{ TNF- $\alpha$} \\
\hline & Mean & SD & Mean & SD & Mean & SD & Mean & SD \\
\hline $\mathrm{NC}$ & 83.14 & 34.51 & 203.12 & $12 \cdot 83$ & $96 \cdot 29$ & 8.63 & $26 \cdot 79$ & 7.62 \\
\hline DSS-C & $298.6^{*}$ & 79.62 & $455 \cdot 86^{*}$ & 101.51 & $255 \cdot 18^{*}$ & 35.6 & $57 \cdot 43^{*}$ & 10.9 \\
\hline DSS-G & 121.65 & 35.98 & $225 \cdot 47$ & $45 \cdot 12$ & $106 \cdot 89$ & 6.47 & 35.08 & $5 \cdot 31$ \\
\hline
\end{tabular}

NC, normal control group; DSS-C, dextran sulphate sodium (DSS)-treated group fed the control diet; DSS-G, DSS-treated group fed the glutamine diet.

${ }^{*}$ Mean values were significantly different from those of the other two groups $(P<0.05)$.

$\dagger$ All the data are representative of duplicate measurements $(n 8)$. Differences among the groups were analysed by a one-way ANOVA with Tukey's multiple-comparison test.

\section{Discussion}

DSS is a sulphated polysaccharide that is commonly used to induce colitis in rodent models. The damage induced by DSS is believed to result from acute chemical toxicity within the colonic epithelium. A recent study has indicated that DSS-induced damage extends to the small intestine by changes in histopathology and an increase in neutrophil activity. Whether the effects of DSS in the small intestine result from a DSS-mediated effect or a compensatory response secondary to colonic changes remains unclear ${ }^{(13)}$. In the present study, we did observe a lower percentage of CD103/ TCR $\gamma \delta^{+} / \mathrm{CD} 8 \alpha \alpha^{+}$T cells belonging to the small-intestinal IEL population after DSS administration and found that Gln administration increased the proportion of small-intestinal IEL $\gamma \delta$-T cells in the DSS-treated mice.

Murine $\gamma \delta$-T cells comprise $5-10 \%$ of the total T-cell population. $\gamma \delta$-IEL are involved in intestinal immunoregulation and serve as the first line of defence against infectious agents ${ }^{(7)}$. Intestinal $\gamma \delta$-T cells produce a variety of cytokines including IFN- $\gamma$, IL- 4 and IL-17 upon bacterial infection ${ }^{(16,17)}$. IL-17 is a cytokine that plays an important role in the orchestration of innate immune function. IL-17 induces pro- and antiinflammatory responses. Although Ogawa et al. ${ }^{(18)}$ have reported that the blockade of IL-17 function by injection of IL-17 antibodies intraperitoneally aggravates DSS-induced colitis, the overexpression of IL-17 has been found to increase neutrophil infiltration, which may result in chronic inflammation and tissue damage ${ }^{(19)}$. Previous studies have shown that the expression of $I L-17$ mRNA is up-regulated in inflamed mucosa. The serum concentrations of IL-17 have also been reported to be elevated in IBD patients ${ }^{(20,21)}$. Complement $\mathrm{C} 5 \mathrm{a}$ is an important proinflammatory mediator and can activate innate immune cells ${ }^{(22)}$. In addition, $\mathrm{C} 5 \mathrm{a}$ has been shown to play an important role in the regulation of the function of $\gamma \delta-T$ cells $^{(23)}$. C 5 a directly acts on the C5aR expressed by $\gamma \delta$-T cells, resulting in the activation of cells, and subsequently enhances their capacity to produce $\mathrm{IL}-17^{(16)}$. Our findings of elevated concentrations of C5aR accompanied by an increased expression of IL-17 in the DSS-C group indicated that small-intestinal IEL $\gamma \delta$-T cells were activated and an inflammatory reaction was initiated in response to DSS. These findings were consistent with the higher concentrations of TNF- $\alpha$ and IFN- $\gamma$ expressed by small-intestinal IEL $\gamma \delta$-T cells in the DSS-C group. TNF- $\alpha$ is an important mediator involved in the onset and regulation of inflammatory and immune responses ${ }^{(24)}$. A previous study has found that IL17-producing $\mathrm{T}$ cells also secrete IFN- $\gamma^{(25)}$. In a study carried out by Tajima et $a l^{(26)}$, naïve $\mathrm{CD}^{+}$cells were transferred into recombination activating gene (RAG)-deficient mice. In that study, it was found that mice with severe colitis had IL-17- and IFN- $\gamma$-double-positive cells in mesenteric lymph nodes. Alternatively, the transfer of naïve $\mathrm{CD}^{+} \mathrm{T}$ cells derived from either $I L-17$ - or $I F N$ - $\gamma$-knockout mice was found to be associated with less severe colitis, indicating that IL-17 and IFN- $\gamma$ can cooperate to cause pathology in this model of colitis. Kuhl et al. ${ }^{(7)}$ also showed that early intestinal $\gamma \delta$-T-cell depletion resulted in the increased production of IFN- $\gamma$ by lamina propria lymphocytes and splenocytes, which may consequently aggravate intestinal inflammation.

KGF, a member of the fibroblast growth factor family, promotes the growth of intestinal epithelial cells. Activated $\gamma \delta$-IEL release KGF near damaged epithelial cells and help maintain and restore the integrity of epithelial tissues after injury $^{(27)}$. A previous study has shown that $K G F$-knockout mice are more susceptible to DSS ${ }^{(7)}$. RegIII $\gamma$ is a bactericidal

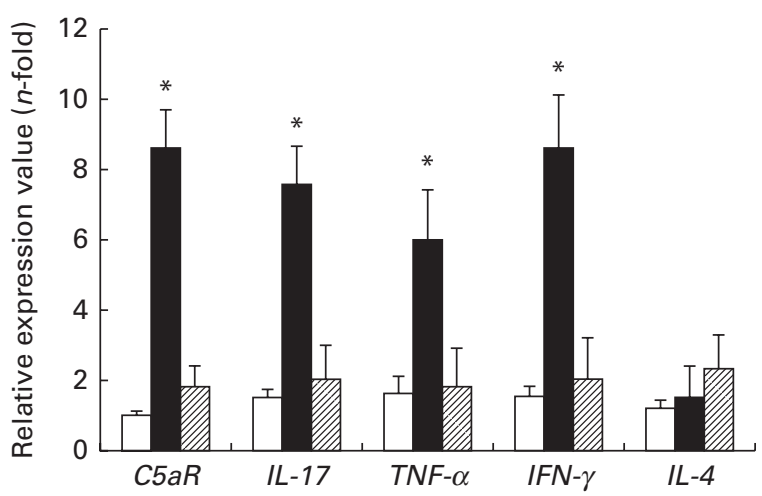

Fig. 2. Expressions of $\mathrm{C} 5 \mathrm{a}$ receptor $(C 5 a R), I L-17, T N F-\alpha$, interferon- $\gamma$ (IFN $\gamma$ ) and IL-4 mRNA in small-intestinal intraepithelial lymphocyte $\gamma \delta-T$ cells. All the data are representative of duplicate measurements $(n 6)$. Values are means, with standard deviations represented by vertical bars. Differences among the groups were analysed by a one-way ANOVA with Tukey's multiple-comparison test. $\square$, Normal control group; $\square$, dextran sulphate sodium (DSS)-treated group fed the control diet; DSS-treated group fed the glutamine diet. *Mean values were significantly different from those of the other two groups $(P<0.05)$. 


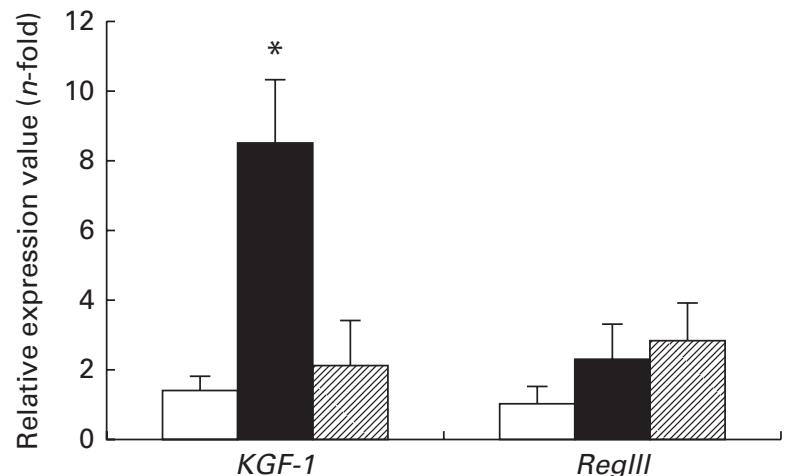

Fig. 3. Expressions of regenerating islet-derived protein III- $\gamma$ (RegIII $\gamma$ ) and keratinocyte growth factor-1 (KGF-1) mRNA in small-intestinal intraepithelial lymphocyte $\gamma \delta-T$ cells. All the data are representative of duplicate measurements $(n 6)$. Values are means, with standard deviations represented by vertical bars. Differences among the groups were analysed by a one-way ANOVA with Tukey's multiple-comparison test. $\square$, Normal control group; $\mathbf{n}$, dextran sulphate sodium (DSS)-treated group fed the control diet; , DSS-treated group fed the glutamine diet. * Mean value was significantly different from those of the other two groups $(P<0.05)$.

lectin secreted into the bowel lumen. A previous study has found that the in vivo depletion of RegIII $\gamma$ from the small intestine decreases bacterial death, whereas mice with recombinant RegIII $\gamma$ have been found to exhibit enhanced small-intestinal bacterial clearance ${ }^{(28)}$. A study carried out by Okamoto \& Sasaki ${ }^{(29)}$ has found that the expression of RegIII mRNA is enhanced in gut epithelial cells of DSS-treated animals and IBD patients. The findings of the present study indicated that the expression of $K G F$ by small-intestinal $\gamma \delta$-T cells was enhanced at the mRNA level in the DSS-treated mice, suggesting that repair mechanisms had been activated. That the expression of RegIII $\gamma$ gene did not differ among the groups suggests that bactericidal activity was not involved in the present experimental conditions.

In the present study, we found that Gln administration had some effects on the responses of small-intestinal IEL $\gamma \delta$-T cells, which were not observed in the DSS-treated mice without Gln supplementation. First, Gln administration reversed the decrease in the percentage of small-intestinal IEL $\gamma \delta$-T cells induced by DSS. Our previous study has also shown that Gln supplementation increases the percentage of smallintestinal IEL $\gamma \delta$-T cells in a septic condition ${ }^{(11)}$. Second, the concentrations of small-intestinal IEL $\gamma \delta$-T-cell-expressed inflammatory mediators, including C5aR, IL-17, TNF- $\alpha$ and IFN- $\gamma$, and inflammatory cytokines secreted into the abdomen were lower in the DSS-G group. These results indicate that small-intestinal inflammation was less severe when Gln was administered. Our findings are consistent with a previous report, which has also found that pretreatment with Gln prevents increases in the expressions of blood IFN- $\gamma$ and IL-17 in acute DSS-induced colitis ${ }^{(30)}$. To better understand the intercellular barrier between epithelial cells, a protein of TJ was studied. TJ form an apical barrier to the paracellular movement of water, solutes and immune cells in polarised epithelium $^{(31)}$. ZO-1 is a membrane phosphoprotein expressed by $\mathrm{TJ}$ of both epithelial and endothelial cells ${ }^{(32)}$. Histological findings of the present study revealed a higher expression of
ZO-1 in the DSS-G group, indicating that the extent of smallintestinal mucosal damage was less severe when Gln was administered. Since the severity of small-intestinal epithelial injury was ameliorated, the expression of the repair gene,

(a)

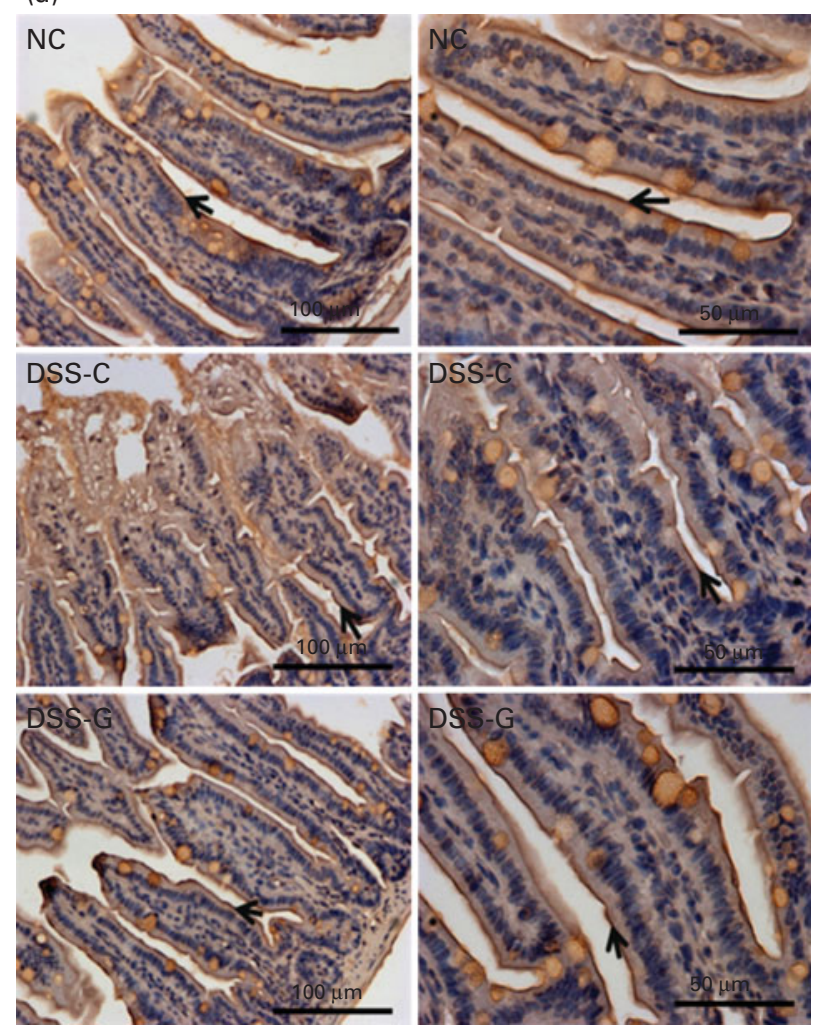

(b)

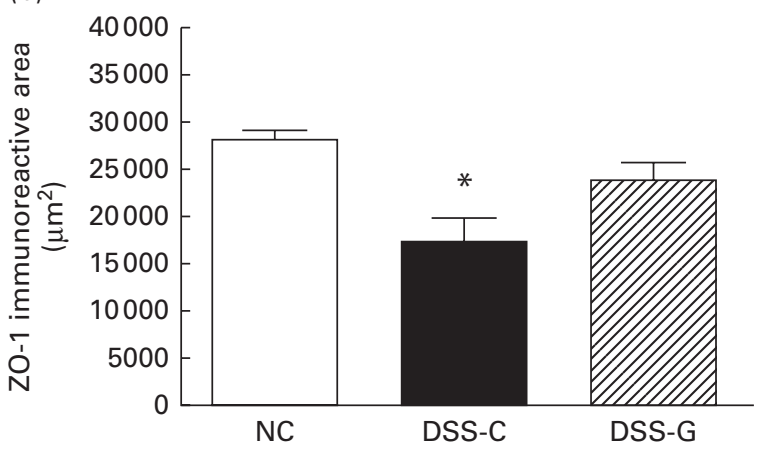

Fig. 4. Distribution of tight junction protein ZO-1-immunoreactive areas in the small-intestinal mucosa of mice in the normal control (NC) group, dextran sulphate sodium (DSS)-treated group fed the control diet group (DSS-C) and DSS-treated group fed the glutamine diet (DSS-G). (a) Representative histological images of mice in the normal control and DSS-treated groups at 100x (left column) and 200x (right column) magnifications. Cell nuclei were contrast-stained with haematoxylin. Arrows indicate ZO-1-positive areas. The normal small intestine exhibited intact epithelium with marked dark-brown ZO-1 expression. In contrast, mucosal structural degeneration and less extensive ZO-1 distribution were observed in the DSS-C group. It is obvious that changes in the DSS-C group were more severe than those in the DSS-G group. (b) Quantification of ZO-1-immunoreactive areas among the groups. The immunoreactive areas of the DSS-G and NC groups were significantly larger than those of the DSS-C group. *Value was significantly different from those of the other two groups $(P<0.05)$. (A colour version of this figure can be found online at http://journals.cambridge.org/bjn). 
$K G F$, may thus have been lowered, as observed in the DSS-G group. A previous study carried out by Nose et al. ${ }^{(10)}$ has demonstrated that Gln administration in mice receiving total parenteral nutrition partially prevents the expression of smallintestinal IEL-derived cytokines, enhances the expression of TJ proteins, and leads to a significant improvement in the function of small-intestinal epithelial barrier.

The beneficial effects of Gln with regard to the attenuation of DSS-induced small-intestinal epithelial injury may involve a complex mechanism. Changes in the expression levels of small-intestinal IEL $\gamma \delta$-T-cell-expressed inflammatory mediators may be only one of several contributory mechanisms. A previous study has found that Gln prevents the activation of NF- $\mathrm{KB}$ and enhances the expression of organ heat shock proteins, thus attenuating organ injury in catabolic conditions ${ }^{(33)}$. In addition, the ability of Gln to reduce oxidative stress may also play a role in the elevation of the expressions of inflammatory mediators. Glutathione is a major antioxidant and acts as a vital component in host defence. Gln has been found to be rate limiting for glutathione synthesis, and the availability of Gln is critical for the generation of glutathione stores ${ }^{(34)}$. However, the mechanism responsible for the protective effect of Gln on small-intestinal epithelial injury requires further investigation.

In summary, the present study has shown for the first time that Gln administration enhances the proportion of $\gamma \delta$-T cells and down-regulates the expression of inflammationassociated mediator genes by small-intestinal IEL $\gamma \delta$-T cells. Histological findings revealed that damage to the small-intestinal mucosa was less severe in the DSS-G group. This result indicates that pretreatment with Gln suppresses the expression of $\mathrm{T}$ helper type $1 / \mathrm{T}$ helper type 17 -associated cytokines by small-intestinal IEL $\gamma \delta$-T cells and may consequently reduce the small-intestinal inflammatory responses, thus ameliorating the severity of DSS-induced small-intestinal epithelial injury.

\section{Acknowledgements}

The present study was supported by a research grant (NSC992320-B-034-002-MY3) from the National Science Council, Taipei, Taiwan. The National Science Council had no role in the design and analysis of the study or in the writing of this article.

The authors' contributions are as follows: M.-H. P. and C.-L. Y. contributed to the concept of the study and carried out most of the data analysis; J.-J. L. carried out part of the analysis; S.-L. Y. and W.-J. C. helped interpret the data; C.-L. Y. and S.-L. Y. prepared the manuscript.

None of the authors has any conflicts of interest.

\section{References}

1. Kaser A, Zeissig S \& Blumberg RS (2010) Inflammatory bowel disease. Annu Rev Immunol 28, 573-621.

2. Maloy KJ \& Powrie F (2011) Intestinal homeostasis and its breakdown in inflammatory bowel disease. Nature $\mathbf{4 7 4}$, 298-306.
3. Cheroutre H, Lambolez F \& Mucida D (2011) The light and dark sides of intestinal intraepithelial lymphocytes. Nat Rev Immunol 11, 445-456.

4. Konkel JE, Maruyama T, Carpenter AC, et al. (2011) Control of the development of CD8alphaalpha + intestinal intraepithelial lymphocytes by TGF-beta. Nat Immunol 12, 312-319.

5. Pardigon N, Darche S, Kelsall B, et al. (2004) The TL MHC class Ib molecule has only marginal effects on the activation, survival and trafficking of mouse small intestinal intraepithelial lymphocytes. Int Immunol 16, 1305-1313.

6. Chen Y, Chou K, Fuchs E, et al. (2002) Protection of the intestinal mucosa by intraepithelial gamma delta $\mathrm{T}$ cells. Proc Natl Acad Sci U S A 99, 14338-14343.

7. Kuhl AA, Pawlowski NN, Grollich K, et al. (2007) Aggravation of intestinal inflammation by depletion/ deficiency of gammadelta $\mathrm{T}$ cells in different types of IBD animal models. J Leukoc Biol 81, 168-175.

8. Wilmore DW \& Shabert JK (1998) Role of glutamine in immunologic responses. Nutrition 14, 618-626.

9. Wischmeyer PE (2007) Glutamine: mode of action in critical illness. Crit Care Med 35, S541-S544.

10. Nose K, Yang H, Sun X, et al. (2010) Glutamine prevents total parenteral nutrition-associated changes to intraepithelial lymphocyte phenotype and function: a potential mechanism for the preservation of epithelial barrier function.J Interferon Cytokine Res 30, 67-80.

11. Lee WY, Hu YM, Ko TL, et al. (2012) Glutamine modulates sepsis-induced changes to intestinal intraepithelial gammadelta T lymphocyte expression in mice. Shock 38, 288-293.

12. Egger B, Bajaj-Elliott M, MacDonald TT, et al. (2000) Characterisation of acute murine dextran sodium sulphate colitis: cytokine profile and dose dependency. Digestion 62 , 240-248.

13. Yazbeck R, Howarth GS, Butler RN, et al. (2011) Biochemical and histological changes in the small intestine of mice with dextran sulfate sodium colitis. J Cell Physiol 226, 3219-3224.

14. Yeh CL, Hsu CS, Yeh SL, et al. (2006) Dietary glutamine supplementation reduces cellular adhesion molecule expression and tissue myeloperoxidase activity in mice with gut-derived sepsis. Nutrition 22, 408-413.

15. Chennupati V, Worbs T, Liu X, et al. (2010) Intra- and intercompartmental movement of gammadelta $\mathrm{T}$ cells: intestinal intraepithelial and peripheral gammadelta $\mathrm{T}$ cells represent exclusive nonoverlapping populations with distinct migration characteristics. J Immunol 185, 5160-5168.

16. Cheng L, Cui Y, Shao H, et al. (2008) Mouse gammadelta $\mathrm{T}$ cells are capable of expressing MHC class II molecules, and of functioning as antigen-presenting cells. J Neuroimmunol 203, 3-11.

17. Inagaki-Ohara K, Sakamoto Y, Dohi T, et al. (2011) $\gamma \delta \mathrm{T}$ cells play a protective role during infection with Nippostrongylus brasiliensis by promoting goblet cell function in the small intestine. Immunology 134, 448-458.

18. Ogawa A, Andoh A, Araki Y, et al. (2004) Neutralization of interleukin-17 aggravates dextran sulfate sodium-induced colitis in mice. Clin Immunol 110, 55-62.

19. Park H, Li Z, Yang XO, et al. (2005) A distinct lineage of CD4 $\mathrm{T}$ cells regulates tissue inflammation by producing interleukin 17. Nat Immunol 6, 1133-1141.

20. Fujino S, Andoh A, Bamba S, et al. (2003) Increased expression of interleukin 17 in inflammatory bowel disease. Gut 52, 65-70.

21. Seiderer J, Elben I, Diegelmann J, et al. (2008) Role of the novel Th17 cytokine IL-17F in inflammatory bowel disease (IBD): upregulated colonic IL-17F expression in active Crohn's disease and analysis of the IL17F p.His161Arg polymorphism in IBD. Inflamm Bowel Dis 14, 437-445. 
22. Peng Q, Li K, Wang N, et al. (2009) Dendritic cell function in allostimulation is modulated by $\mathrm{C} 5 \mathrm{aR}$ signaling. J Immunol 183, 6058-6068.

23. Han G, Geng S, Li Y, et al. (2011) $\gamma \delta$ T-cell function in sepsis is modulated by $\mathrm{C} 5$ a receptor signalling. Immunology $\mathbf{1 3 3}$, 340-349.

24. Beutler B \& Cerami A (1988) Tumor necrosis, cachexia, shock, and inflammation: a common mediator. Annu Rev Biochem 57, 505-518.

25. Annunziato F, Cosmi L, Santarlasci V, et al. (2007) Phenotypic and functional features of human Th17 cells. J Exp Med 204, 1849-1861.

26. Tajima M, Wakita D, Noguchi D, et al. (2008) IL-6-dependent spontaneous proliferation is required for the induction of colitogenic IL-17-producing $\mathrm{CD}^{+}$T cells. J Exp Med 205, 1019-1027.

27. Chen EJ \& Kaiser CA (2002) Amino acids regulate the intracellular trafficking of the general amino acid permease of Saccharomyces cerevisiae. Proc Natl Acad Sci US A 99, 14837-14842.

28. Brandl K, Plitas G, Schnabl B, et al. (2007) MyD88-mediated signals induce the bactericidal lectin RegIII gamma and protect mice against intestinal Listeria monocytogenes infection. J Exp Med 204, 1891-1900.
29. Okamoto H \& Sasaki I (2003) Increased expression of HIP/ $P A P$ and regenerating gene $I I I$ in human inflammatory bowel disease and a murine bacterial reconstitution model. Inflamm Bowel Dis 9, 162-170.

30. Chu CC, Hou YC, Pai MH, et al. (2012) Pretreatment with alanyl-glutamine suppresses T-helper-cell-associated cytokine expression and reduces inflammatory responses in mice with acute DSS-induced colitis. J Nutr Biochem 23, 1092-1099.

31. Fanning AS, Jameson BJ, Jesaitis LA, et al. (1998) The tight junction protein ZO-1 establishes a link between the transmembrane protein occludin and the actin cytoskeleton. J Biol Chem 273, 29745-29753.

32. Willott E, Balda MS, Fanning AS, et al. (1993) The tight junction protein ZO-1 is homologous to the Drosophila discs-large tumor suppressor protein of septate junctions. Proc Natl Acad Sci U S A 90, 7834-7838.

33. Singleton KD \& Wischmeyer PE (2007) Glutamine's protection against sepsis and lung injury is dependent on heat shock protein 70 expression. Am J Physiol Regul Integr Comp Physiol 292, R1839-R1845.

34. Welbourne TC (1979) Ammonia production and glutamine incorporation into glutathione in the functioning rat kidney. Can J Biochem 57, 233-237. 